\title{
Idiopathic renal hypouricemia: A case report and literature review
}

\author{
CUIYU WANG ${ }^{1}$, JIN WANG $^{2}$, SONG LIU $^{3}$, XINHUA LIANG $^{1}$, YIFAN SONG ${ }^{1}$, \\ LING FENG $^{1}$, LANXIN ZHONG ${ }^{1}$ and XIAOHUA GUO ${ }^{1}$ \\ ${ }^{1}$ Department of Nephrology, Shenzhen Hospital, Southern Medical University, Shenzhen, Guangdong 518000; \\ ${ }^{2}$ Department of Epidemiology, School of Public Health, Sun Yat-Sen University, Guangzhou Guangdong 510080; \\ ${ }^{3}$ Department of Nephrology, Dalian Liguang Rehabilitation Hospital, Dalian, Liaoning 116000, P.R. China
}

Received November 3, 2018; Accepted August 20, 2019

DOI: $10.3892 / \mathrm{mmr} .2019 .10726$

\begin{abstract}
Idiopathic renal hypouricemia is a rare hereditary condition. Type 2 renal hyperuricemia (RHUC2) is caused by a mutation in the SLC2A9 gene, which encodes a high-capacity glucose and urate transporter, glucose transporter (GLUT) 9. RHUC2 predisposes to exercise-induced acute renal failure (EIARF) and nephrolithiasis, which is caused by a defect in renal tubular urate transport and is characterized by increased clearance of renal uric acid. In the present study a case of a 35-year-old Chinese man with EIARF is reported. The patient had isolated renal hypouricemia, with a serum uric acid level of $21 \mu \mathrm{mol} / 1$ and a fractional excretion of uric acid of $200 \%$. The mutational analysis revealed a homozygous mutation (c.857G $>$ A in exon 8 ) in the SLC2A9 gene. The patient's family members carried the same mutation, but were heterozygous and clinically asymptomatic. In conclusion, to the best of our knowledge, this is the first report of a RHUC2 patient with a GLUT9 mutation, $p . W 286 X$, which may be a pathogenic mutation of RHUC2. Further investigation into the functional role of GLUT9 in this novel SLC2A9 mutation is required.
\end{abstract}

\section{Introduction}

Hypouricemia is defined as a serum urate concentration of $<119 \mu \mathrm{mol} / \mathrm{l}$ ( $2 \mathrm{mg} / \mathrm{dl})$. Hypouricemia may be due to decreased uric acid production, defective renal tubular reabsorption caused by inherited or acquired disorders, or uric acid oxidation caused by treatment with uricase (1). The kidney is an important regulator of uric acid homeostasis, as urinary uric acid excretion normally accounts for $2 / 3$ of the daily uric acid clearance. The uric metabolism is consistent with a four-step model for renal urate handling, comprising glomerular filtration, pre-secretion reabsorption, secretion and post-secretion

Correspondence to: Dr Xiaohua Guo, Department of Nephrology, Shenzhen Hospital, Southern Medical University, 1333 Lake Road, Shenzhen, Guangdong 518000, P.R. China

E-mail: 632583102@qq.com

Key words: idiopathic renal hypouricemia, exercise-induced acute renal failure, gene analysis, $S L C 2 A 9$ mutations reabsorption (2), with the latter three steps occurring in the proximal tubules, where urate is bidirectionally transported. As a result, $\sim 10 \%$ of the filtered load of urate is excreted in the urine (3).

Idiopathic renal hypouricemia (iRHUC) is a rare hereditary disease caused by impaired uric acid transport, reabsorption insufficiency and/or secretion acceleration $(4,5)$. There are two types of RHUC: Type 1 (RHUC1), which is caused by a mutation in the $S L C 22 A 12$ gene that encodes a renal urate-anion exchanger, URAT1 (4,5); whereas type 2 (RHUC2) was previously found to be caused by a defect in the SLC $2 A 9$ gene, which encodes a high-capacity glucose and urate transporter, named glucose transporter (GLUT)9 (6). The majority of patients with iRHUC are clinically asymptomatic. However, patients with homozygous SLC2A9 mutations may present with nephrolithiasis, hematuria or exercise-induced acute kidney injury (EIAKI) (7), accompanied by homozygous loss-of-function mutations of GLUT9 and a resultant total defect of uric acid absorption.

The diagnosis of iRHUC is based on hypouricemia $(<119 \mu \mathrm{mol} / 1$ or $2.0 \mathrm{mg} / \mathrm{dl})$ and increased fractional excretion of uric acid (FE-UA) of $>10 \%$, without evidence of secondary causes of hypouricemia. The diagnosis can be confirmed by molecular analysis of the mutations in the SCL22A12 and/or SLC2A9 genes.

Over 100 cases with $S L C 22 A 12$ mutations and 20 cases with SLC2A9 defects, summarized in a Chinese literature review (8), have been reported to date worldwide. These patients exhibited common characteristics, including affected family members with inherited renal tubular defects resulting in hypouricemia, increased urinary excretion of urate, susceptibility to EIAKI and chronic renal dysfunction (9). The present study describes the case of a patient with iRHUC who presented with EIAKI and had a homozygous mutation c. $857 \mathrm{G}>\mathrm{A}$ in exon 8 of the SLC $2 A 9$ gene.

\section{Case report}

A 35-year-old young man with unremarkable medical history was admitted to the Nephrology Department of Shenzhen Hospital, Southern Medical University on May 23, 2018. The patient complained of nausea, vomiting and abdominal pain for 3 days after strenuous exercise, but without oliguria, hematuria or myalgia. The physical examination was performed and vital signs were normal. The laboratory tests revealed increased 
Table I. Laboratory results of the proband.

\begin{tabular}{|c|c|c|c|c|c|}
\hline Parameter & Day $^{\mathrm{a}} 5.23$ & Day 5.31 & Day 6.02 & Day $^{\mathrm{b}} 6.15$ & Day 7.16 \\
\hline Urea (mg/dl) & 67.76 & 40.6 & 37.52 & 21.8 & 12.3 \\
\hline Creatinine (mg/dl) & 11.86 & 8.59 & 6.40 & 2.22 & 1.20 \\
\hline Uric acid (umol/l) & 265 & 105 & 44.5 & 21 & 18 \\
\hline Sodium (mmol/l) & 138.2 & 139.6 & & 142.2 & 141.5 \\
\hline Potassium (mmol/l) & 3.84 & 4.39 & & 4.19 & 3.92 \\
\hline Calcium (mmol/l) & 1.98 & 2.18 & & 2.4 & 2.42 \\
\hline Phosphorus (mmol/l) & 2.24 & & & 1.15 & 1.10 \\
\hline Albumin $(\mathrm{g} / \mathrm{l})$ & 41.2 & & & 45.6 & 48.7 \\
\hline $\operatorname{ALT}(\mathrm{U} / \mathrm{l})$ & 36 & & & 35 & 65 \\
\hline AST (U/l) & 26 & & & 24 & 37 \\
\hline PTH (pg/ml) & 310.1 & & & 87.49 & \\
\hline
\end{tabular}

Serum urate levels $(1 \mathrm{mg} / \mathrm{dl}$ is equivalent to $59.5 \mathrm{umol} / \mathrm{l})$; Serum urate levels normal range: Man: $149-416 \mu \mathrm{mol} / \mathrm{l}(2.5-7.0 \mathrm{mg} / \mathrm{dl})$; woman: 89-357 $\mu \mathrm{mol} / 1$ (1.5-6.0 mg/dl). ${ }^{\mathrm{a} I n i t i a t i o n}$ of dialysis. ${ }^{\mathrm{b}}$ Day of renal biopsy. ALT, alanine aminotransferase; AST, aspartate aminotransferase; PTH, parathyroid hormone.

levels of urea $(67.76 \mathrm{mg} / \mathrm{dl})$ and creatinine $(11.86 \mathrm{mg} / \mathrm{dl})$, but a normal uric acid level at $265 \mu \mathrm{mol} / 1(1 \mathrm{mg}=59.5 \mu \mathrm{mol} / \mathrm{l}$; Table I). Therefore, the patient was diagnosed with AKI and was started on hemodialysis.

A total of two family members of the patient had uremia (Fig. 1) and required long-term renal replacement therapy, but without any evident causes of renal insufficiency. The patient's family history included consanguineous parents (cousins), whereas both parents had nephrolithiasis; the proband had one healthy brother. On serum UA level screening, the proband's parents, younger brother and son all had normal serum urate levels.

FE-UA was $200 \%$ of the normal reference range of 8.3 (5.5-11.1)\%. Renal ultrasound revealed hyperechogenic kidneys, without detection of stones (Fig. 2). Due to the deterioration of renal function, the patient received hemodialysis treatment for 2 weeks, starting on the 2 nd day after hospitalization; subsequently, a renal biopsy was performed.

Histological analysis (Fig. 3) revealed normal glomeruli and arterioles, patchy or diffuse denudation and vacuolar degeneration in the renal tubular cells with loss of the brush border, accompanied by interstitial edema. Immunofluorescence showed traces of C3; however, C4, immunoglobulin (Ig)A, IgG, IgM and fibrinogen (Fib) were all negative.

The patient was advised to avoid physical exertion and increase his fluid intake. One month after his discharge from the hospital, his uric acid level was $18 \mu \mathrm{mol} / \mathrm{l}$, with improved renal function (blood urea nitrogen level: $12.3 \mathrm{mg} / \mathrm{dl}$ and serum creatinine: $1.2 \mathrm{mg} / \mathrm{dl}$; Table I), suggesting recovery of the kidneys from acute tubular necrosis (ATN).

A SLC $2 A 9$ homozygous mutation was identified (Figs. 4 and 5), namely 857G $>$ A ( $p . W 286 X$; nucleotide number 857 in the coding region is mutated from guanine to adenine), resulting in amino acid changes. The variation in the normal population database frequency is 0.00020 . It was verified that the patient's family (his father, mother, younger brother and son) were heterozygous for this site, which is a suspected pathogenic mutation (Table II).

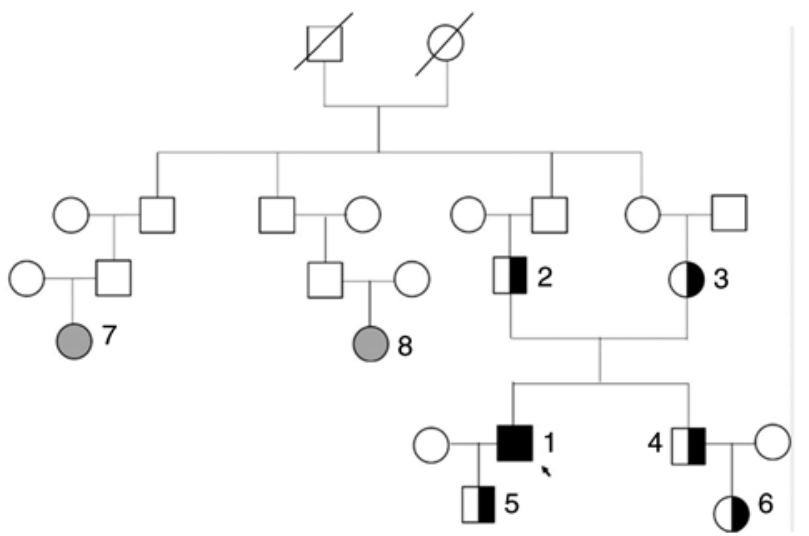

Figure 1. Pedigrees of consanguineous families with severe renal hypouricemia and SLC2A9 mutations. Solid symbols denote affected family member, gray symbols denote end-stage renal disease patients, white squares healthy members and unexamined family members, half-solid denote heterozygous family members. Circles represent female family members, squares represent male family members and crosses represent dead family members. The results of the analysis in the patient's family members suggested an autosomal recessive mode of inheritance. The proband is marked by an arrow.

Sequence analysis of the SLC22A12 and SLC2A9 genes was performed in the patient and his family members (his father, mother, brother, son and nephew) by Beijing MyGenostics, Co., Ltd. Briefly, the detection process was as follows: i) DNA extraction and next generation sequencing library preparation: Genomic DNA was extracted from whole blood using the QIAamp DNA Mini kit (180134; Qiagen, Inc.) following the manufacturer's protocol. ii) Targeted gene capture: Next, genes associated with renal hypouricemia and other hereditary nephropathy-related diseases were selected by a gene capture strategy, using the GenCap custom enrichment kit (MyGenostics Inc.) following the manufacturer's protocol. iii) Sequencing: The enriched libraries were sequenced on an Illumina HiSeq 2000 sequencer (Illumina, Inc.) for paired-end reads of $150 \mathrm{bp}$. iv) Data analysis and determination of gene pathogenicity. 

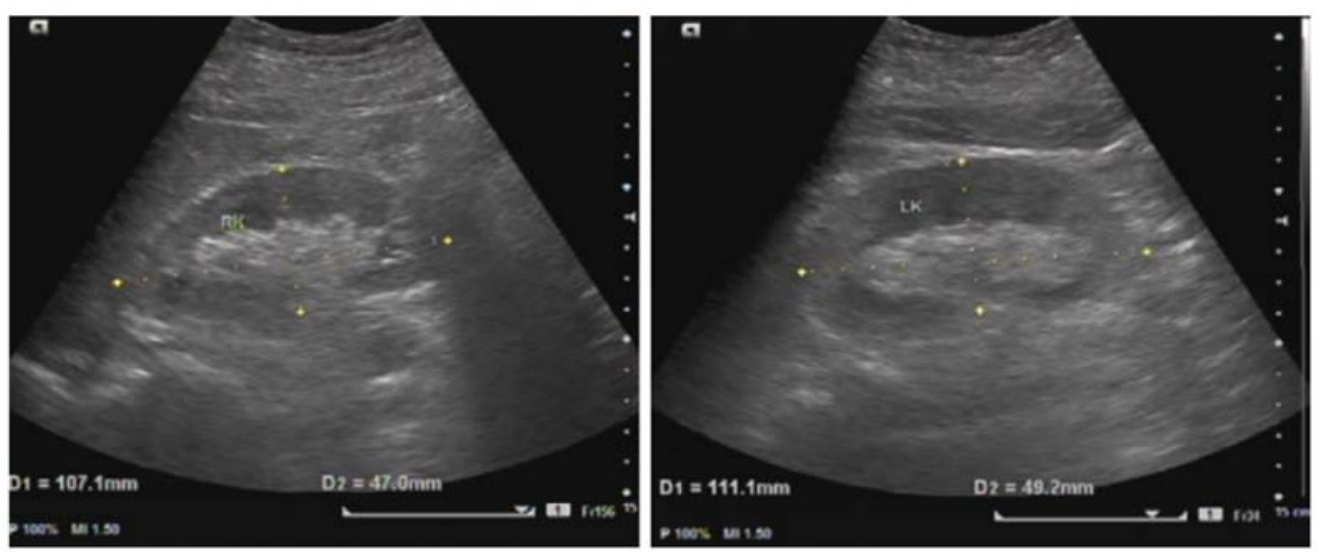

Figure 2. Ultrasound of a slightly hyperechogenic renal cortex and kidneys with no stones.
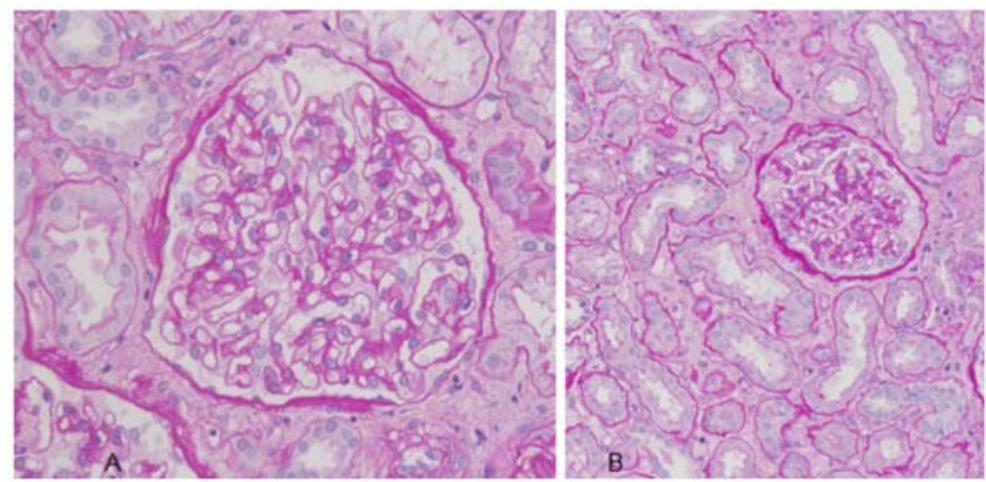

Figure 3. A renal biopsy is performed 3 weeks after admission. The light microscope revealed very slight mesangial cell proliferation and normal arterioles, with vacuolar degeneration in the tubular epithelial cells, accompanied by interstitial edema, scattered lymphocytes and monocyte infiltration. Immunofluorescence staining for IgG, IgA, IgM, C3, C4 and Fib were all negative. (A) Magnification, x400 and (B) magnification x200. Ig, immunoglobulin.

v) Validation by Sanger Sequencing: All mutations identified by HiSeq 2000 sequencing were confirmed by Sanger sequencing.

\section{Discussion}

Increased urinary excretion of uric acid may be observed in patients with familial hypouricemia (an inherited disorder) and in association with a variety of acquired conditions. Hereditary hypouricemia may be complicated by nephrolithiasis and EIARF. A total of two types of uric acid transport proteins, uric acid transporter 1 (URAT1) and GLUT9, expressed in the gut as well as the kidney, regulate serum urate levels (4-6). Previous studies have reported cases of patients with hypouricemia due to loss-of-function mutations of the URAT1 gene $(4,9)$. Furthermore, several studies also reported that loss-of-function mutations in the SLC2A9 gene (encoding GLUT9) also cause hypouricemia $(6-8,10)$.

Familial hypouricemia, also referred to as iRHU, is caused by a defect in renal tubular urate transport. The majority of iRHU cases are caused by a mutation in the SLC22A12 gene that encodes URAT1, among which $\sim$ half are homozygotes, one-third compound heterozygotes and the remaining cases are heterozygotes (11). In Japanese and Koreans, the W258X mutation is reported as the predominant genetic cause of iRHU $(3,11)$. URAT1 is highly urate-specific and it is expressed in the luminal membrane of the proximal tubular cells, but is absent from the distal tubular cells or elsewhere in the body; it is largely responsible for proximal urate reabsorption (Fig. 6).

The residual apical uptake of urate is likely mediated by the OAT4 (SLC22A11) and OAT10 (SLC22A13) urate-anion exchangers $(12,13)$. Mutations in the $S L C 2 A 9$ gene have also been found to be associated with familial renal hypouricemia. This gene encodes the high-capacity urate transporter GLUT9. GLUT9 has two subtypes, one short (GLUT9S) and one long $(G L U T 9 L)(6,7,10)$. Urate reabsorption from the tubular lumen into the cell is mediated by URATI and other anion exchangers, as mentioned above. Uric acid efflux from the cell across the basal membrane appears to be mediated only by basolateral GLUT9a (14) (Fig. 6). There have been several reports of $S L C 2 A 9$ gene mutations leading to iRHU. One study (7) reported that the impact of GLUT9 deficiency on renal excretion of uric acid and serum uric acid levels exceeds that of URATl deficiency. In the present study, the result of the genetic test revealed homozygosity for a $S L C 2 A 9$ mutation, the source of which were the parents. The uric acid excretion rate was as high as $200 \%$. The result is consistent with the report (7), of a girl with severe iRHUC (serum urate $2.97 \mu \mathrm{mol} / 1$, fractional excretion of uric acid 295.99\%).

The SLC2A9 gene is located on human chromosome 4p15.3-16, including 14 exons ( 1 non-coding and 13 coding), and is $195-\mathrm{Kb}$ long, encoding 540 amino acids. There have been a number of studies on SLC2A9 mutations leading to 
$\begin{array}{llllllllllllllllllllllllll}\bar{T} & \bar{A} & \bar{C} & \bar{G} & \bar{T} & \bar{C} & \bar{C} & \bar{G} & \bar{C} & \bar{T} & \bar{A} & \bar{G} & \bar{C} & \bar{A} & \bar{G} & \bar{G} & \bar{T} & \bar{G} & \bar{G} & \bar{T} & \bar{C}\end{array}$

Patient

/Hom

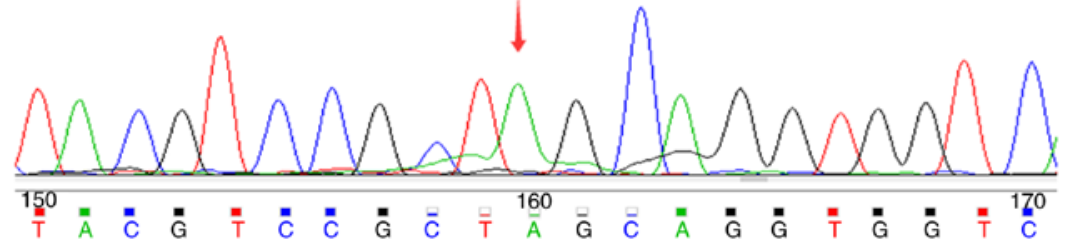

His father

/Het

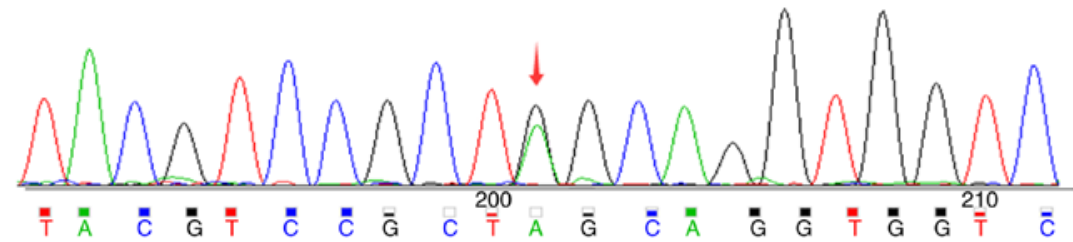

His mother

/Het

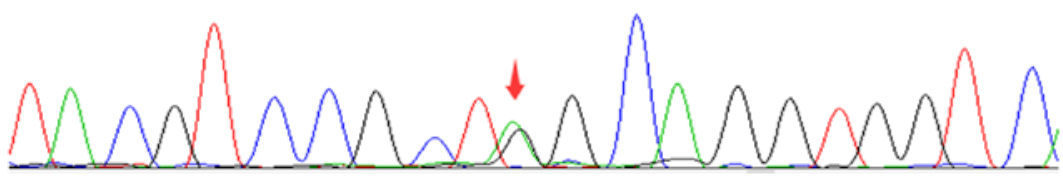

Figure 4. A SLC2A9 homozygous mutation of the patient was identified, namely $857 \mathrm{G}>\mathrm{A}$. The father and mother were heterozygous. Hom/het: Hom indicates that the mutation site is a homozygous mutation, het indicates that the mutation site is a heterozygous mutation.

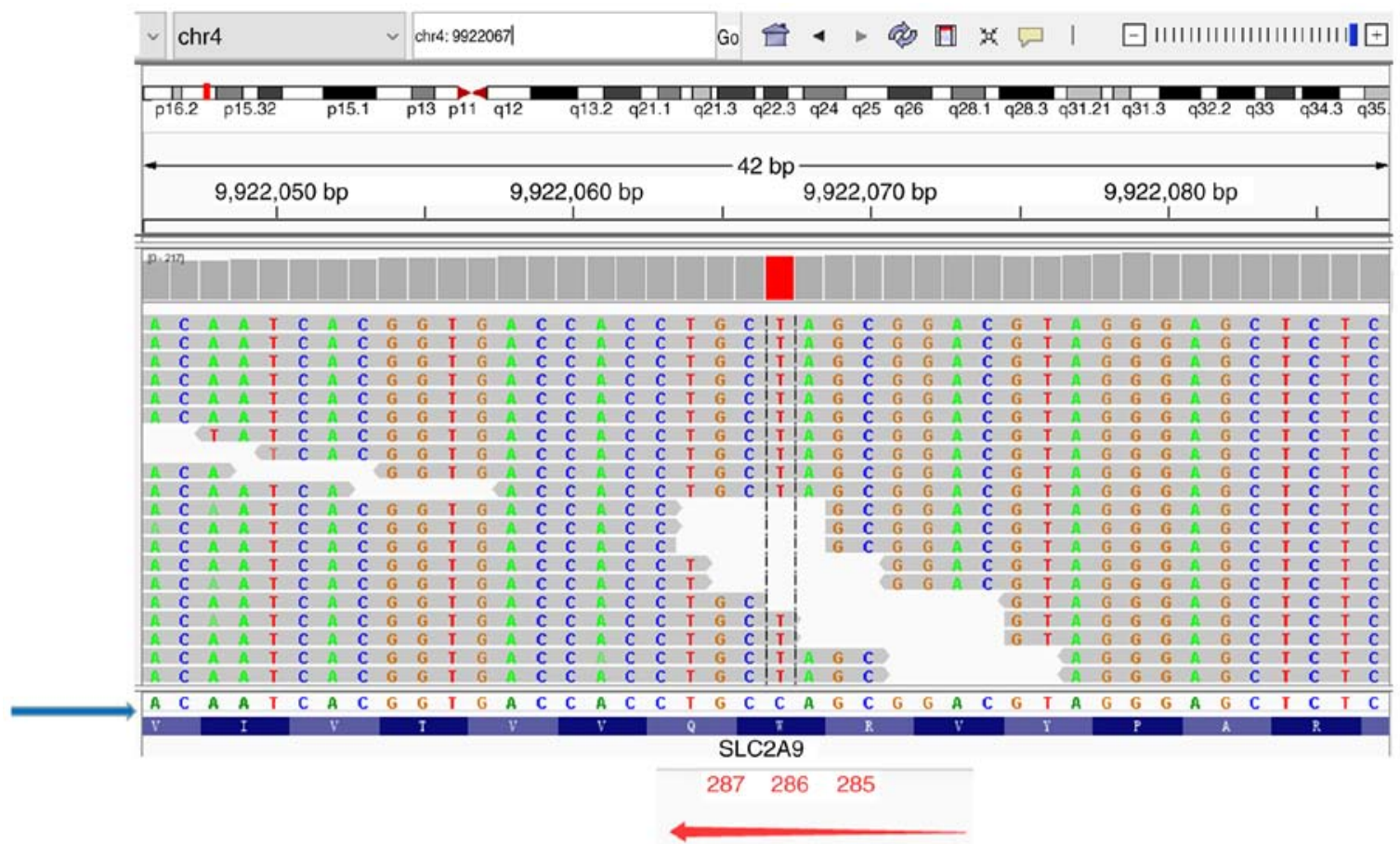

Figure 5. Gene sequence indicated by the blue arrow is the reference sequence of the SLC2A9 gene. Below the reference gene sequence are the encoded amino acid sequence. It shows three amino acid sequence at the sites of 285,286 and 287 . The gene mutation site of the patient is 286 . The gene coding direction is from the right to the left (the direction indicated by the red arrow).

low uric acid nephropathy, but the gene mutation sites are different. Several Japanese studies have investigated hypouricemic acid nephropathy and reported a number of cases, among which two families were found to have RHUC2 due to GLUT9 missense mutations $R 198 C$ or $R 380 W(10,15)$. A total of two Chinese studies reported a homozygous mutation (g.68G $>\mathrm{A}$ in exon 3 ) in the $S L C 2 A 9$ gene (8) and a homozygous splice-site mutation $(\mathrm{c} .1215+1 \mathrm{G}>\mathrm{A})$ in 
GLUT9L (16), corresponding to c. $1128+1 \mathrm{G}>\mathrm{A}$ in $G L U T 9 S)$. In Spanish patients (17), the $S L C 2 A 9$ mutation site was reported to be p.T125M. A young Pakistani patient (18) was reported to have severe renal hypouricemia, with compound heterozygosity for SLC2A9 p.Arg380Trp and p.Gly216Arg mutations. A total of two British pediatric patients (19) with AKI were found to have the missense transitions p.G216R and p.N333S in the SLC2A9 gene. The majority of reported pathogenic $S L C 2 A 9$ gene mutations are homozygous, but heterogeneous mutations of the $S L C 2 A 9$ gene may also lead to RHUC2 $(13,15)$.

In the present case, a novel homozygous mutation, c. $857 \mathrm{G}>\mathrm{A}(p . W 286 X)$, was identified in exon 8 of $S L C 2 A 9$ (Figs. 4 and 5). This mutation leads to amino acid changes. Whether these specific amino acid changes compromised the uric acid transport by GLUT9 remains elusive. To the best of our knowledge, mutations at this site have not been reported in previous genetic studies on hypouricemic acid nephropathy. The prediction results of protein function prediction software SIFT (http://sift.jcvi.org/), PolyPhen_2 (http://genetics. bwh.harvard.edu/pph2/) and REVEL (https://sites.google. $\mathrm{com} / \mathrm{site} / \mathrm{revelgenomics/downloads)} \mathrm{all} \mathrm{showed} \mathrm{'unknown'.}$ Therefore, the significance of the amino acid substitution in the novel SLC2A9 mutation needs be determined.

EIARF has been reported in patients with familial renal hypouricemia $(9,20)$. It was first reported in 1989 by Erley et al (21). The largest study to date was a review of 54 patients with renal hypouricemia, of whom $~ 90 \%$ were male (20). AKI most often occurs after strenuous exercise, such as a short-distance race. The presenting symptoms are always severe abdominal pain and nausea, usually occurring within 6-12 $\mathrm{h}$ after exercise. At the time of presentation, the mean serum creatinine level of the patients was $5.5 \mathrm{mg} / \mathrm{dl}$ $(486 \mathrm{mmol} / \mathrm{l})$ and the mean serum uric acid level was normal (262 $\mu \mathrm{mol} / \mathrm{l})$, which was at least partly indicative of be renal failure. After recovery, serum uric acid was reduced to $42 \mu \mathrm{mol} / 1$. Renal function was restored in all patients, whereas some patients required hemodialysis. During follow-up, 13 patients (24\%) developed recurrent AKI; repeated AKI episodes may lead to chronic kidney disease in some patients (20).

The mechanisms underlying renal hypouricemia-induced EIAKI remain unclear. Two possible pathogenetic mechanisms have been proposed: i) During exercise, increased oxidative stress may lead to renal vasoconstriction, ischemia and oxidative damage (22), possibly leading to reduced glomerular filtration rate and acute tubular injury (also referred to as ATN). ii) During exercise, increased uric acid production leads to uric acid excretion stress and deposition in the renal tubules, as occurs in uric acid nephropathy.

For patients with renal hypouricemia-related AKI, most of the reported renal biopsy results showed ATN, without renal uric acid crystallization and intratubular deposition $(20,23)$. Plasma uric acid is a powerful antioxidant, which appears to play a protective role in the kidney; renal hypouricemia may be associated with decreased antioxidant capacity and potential kidney injury caused by reactive oxygen species (24). It is hypothesized that a decrease in circulating uric acid, a known antioxidant (25), impairs the ability of the kidney to respond to increased oxidative stress associated with strenuous 


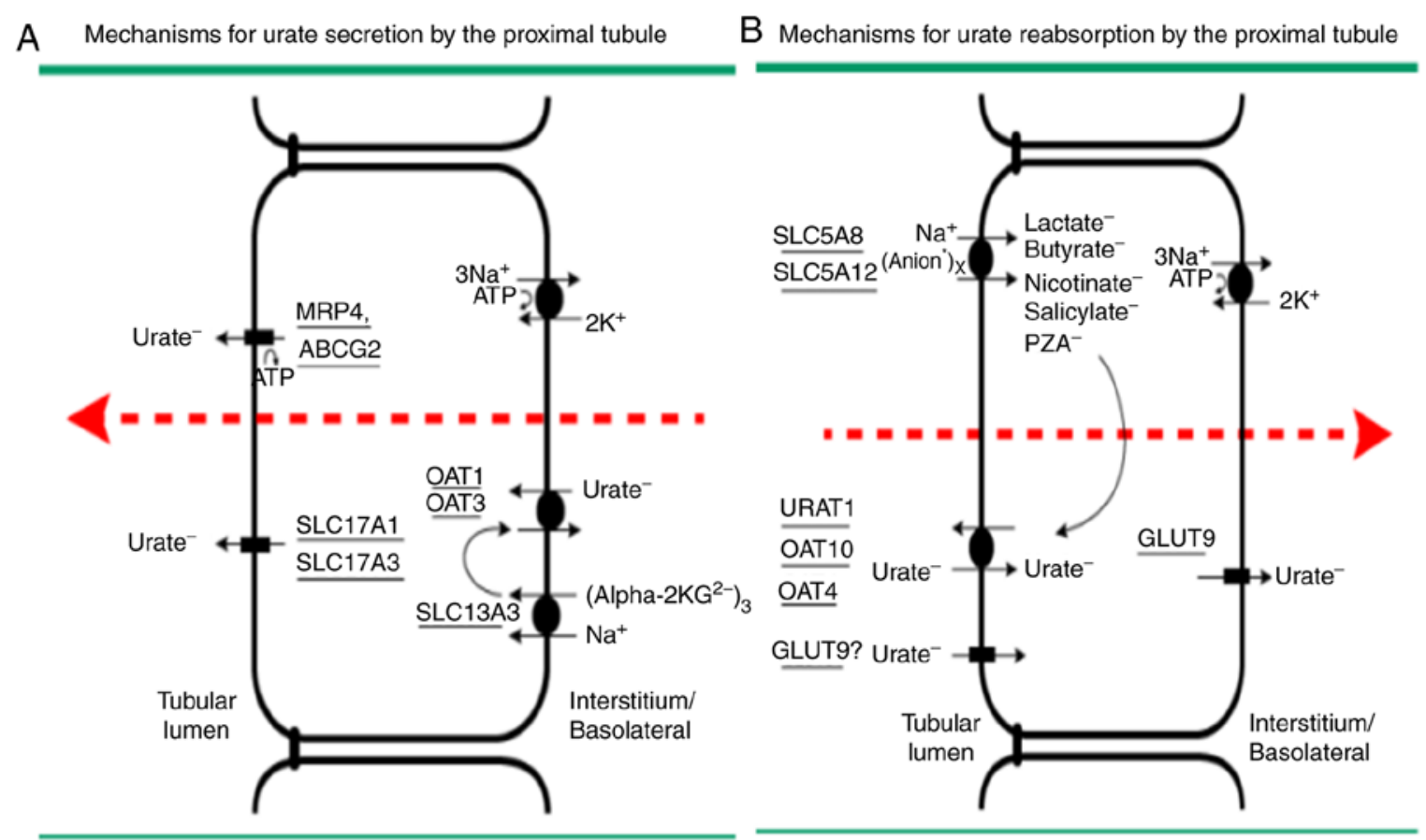

Figure 6. Mechanisms for urate secretion and reabsorption by the proximal tubule. (A) Urate enters the cell at the basolateral membrane via exchange with $\alpha$-ketoglutarate, mediated by OAT1 and OAT3. At the apical membrane, urate is secreted via MRP4, ABCG2, NPT1 (SLC17A1) and/or NPT4 (SLC17A3). SLC13A1 is a sodium-dependent transporter that drives basolateral urate uptake. (B) Sodium-dependent anion transport by SLC5A8 and SLC5A12 increases intracellular concentrations of anions that exchange with luminal urate (URAT1/OAT10/OAT4). GLUT9 is the presumptive exit pathway for urate at the basolateral membrane but may also traffic to the apical membrane. Both images A and B were produced with up to date research. OAT1, Solute carrier family 22 member 6; OAT3, Solute carrier family 22 member 8; MRP4, multidrug resistance-associated protein 4; ABCG2, ATP-binding cassette sub-family G member 2; SLC17A1, sodium-dependent phosphate transport protein 1; GLUT9, Solute carrier family 2, facilitated glucose transporter member 9; URAT1, Solute carrier family 22 member 12 .

exercise (26). The pathological examination of renal biopsy samples supports this hypothesis.

The following conditions support the theory of uric acid deposition: In a case report (21) on EIAKI, a renal biopsy revealed blockage of the renal tubules by uric acid crystals; in addition, an increased prevalence of uric acid kidney stones has been reported in patients with renal hypouricemia $(7,27,28)$. A total of 19 patients with familial renal hypouricemia were enrolled in two case series studies, of whom 5 (26\%) had a history of kidney stones $(27,28)$. The majority of these reports describe uric acid stones (7). However, the pathological examination of renal biopsy samples does not support this view. Further evidence comes from patients with recurrent EIAKI that may be preventable by allopurinol therapy. Bhasin et al (29) reported that an 18-year-old male patient repeatedly developed AKI after a 400-m race and was eventually diagnosed with iRHUC. The patient was prescribed oral allopurinol tablets $300 \mathrm{mg} /$ day x 3 days and AKI did not develop again after the race. However, allopurinol is also an antioxidant, which may partly explain its protective effect against AKI (22). Therefore, the mechanism of EIARF remains unclear and requires further research.

In conclusion, this is the first report of a patient with RHUC2 due to the mutation of SLC2A9, which encodes GLUT9. p.W286X may be a pathogenic mutation of RHUC2; however, further investigations into the functional properties of GLUT9 in this novel $S L C 2 A 9$ mutation are required. In clinical practice, the diagnosis of EIAKI should be considered in patients manifesting symptoms of AKI and moderately elevated or normal serum concentrations of uric acid, particularly after strenuous exercise.

\section{Acknowledgements}

Not applicable.

\section{Funding}

No funding was received.

\section{Availability of data and materials}

The datasets used and/or analyzed during the present study are available from the corresponding author on reasonable request.

\section{Authors' contributions}

XHG designed the study and guided the writing of the manuscript. CYW drafted the manuscript and oversaw the figures. JW helped in drawing Fig. 1, analyzing and discussing the results of Figs. 4 and 5, and describing the pathogenic SLC22A9 mutation in detail. SL contributed to the collection of important background information and editing the language (translating Chinese into English). XHL and YFS performed the histological examination and completed the manuscript review. LF and LXZ provided and collated medical records. 


\section{Ethics approval and consent to participate}

The Institutional Ethical Committee approved the publication of material relating to the patient and his family in domestic and international academic journals.

\section{Patient consent for publication}

Informed consent was obtained from the patient regarding the publication of the case details.

\section{Competing interests}

The authors declare that they have no competing interests.

\section{References}

1. Maesaka JK and Fishbane S: Regulation of renal urate excretion: A critical review. Am J Kidney Dis 32: 917-933, 1998.

2. Roch-Ramel F and Diezi J: Renal transport of organic ions and uric acid. In: Diseases of the kidney. Schrier RW and Gottschalk CE (eds). 6th edition. Little, Brown and Co., Boston, pp231-249, 1997.

3. Komoda F, Sekine T, Inatomi J, Enomoto A, Endou H, Ota T, Matsuyama T, Ogata T, Ikeda M, Awazu M, et al: The W258X mutation in SLC22A12 is the predominant cause of Japanese renal hypouricemia. Pediatr Nephrol 19: 728-733, 2004.

4. Enomoto A, Kimura H, Chairoungdua A, Shigeta Y, Jutabha P, Cha SH, Hosoyamada M, Takeda M, Sekine T, Igarashi T, et al: Molecular identification of a renal urate anion exchanger that regulates blood urate levels. Nature 417: 447-452, 2002.

5. Stiburkova B, Sebesta I, Ichida K, Nakamura M, Hulkova H, Krylov V, Kryspinova L and Jahnova H: Novel allelic variants and evidence for a prevalent mutation in URAT1 causing renal hypouricemia: Biochemical, genetics and functional analysis. Eur J Hum Genet 21: 1067-1073, 2013.

6. Vitart V, Rudan I, Hayward C, Gray NK, Floyd J, Palmer CN, Knott SA, Kolcic I, Polasek O, Graessler J, et al: SLC2A9 is a newly identified urate transporter influencing serum urate concentration, urate excretion and gout. Nat Genet 40: 437-442, 2008.

7. Dinour D, Gray NK, Campbell S, Shu X, Sawyer L, Richardson W, Rechavi G, Amariglio N, Ganon L, Sela BA, et al: Homozygous SLC2A9 mutations cause severe renal hypouricemia. J Am Soc Nephrol 21: 64-72, 2010.

8. Shen H, Feng C, Jin X, Mao J, Fu H, Gu W, Liu A, Shu Q and Du L: Recurrent exercise-induced acute kidney injury by idiopathic renal hypouricemia with a novel mutation in the SLC2A9 gene and literature review. BMC Pediatr 14: 73, 2014.

9. Ichida K, Hosoyamada M, Hisatome I, Enomoto A, Hikita M, Endou $\mathrm{H}$ and Hosoya T: Clinical and molecular analysis of patients with renal hypouricemia in Japan-influence of URAT1 gene on urinary urate excretion. J Am Soc Nephrol 15: 164-173, 2004.

10. Matsuo H, Chiba T, Nagamori S, Nakayama A, Domoto H, Phetdee K, Wiriyasermkul P, Kikuchi Y, Oda T, Nishiyama J, et al: Mutations in glucose transporter 9 gene SLC2A9 cause renal hypouricemia. Am J Hum Genet 83: 744-751, 2008.

11. Cheong HI, Kang JH, Lee JH, Ha IS, Kim S, Ha IS, Kim S, Komoda F, Sekine T, Igarashi T and Choi Y: Mutational analysis of idiopathic renal hypouricemia in Korea. Pediatr Nephrol 20: 886-890, 2005.

12. Hagos Y, Stein D, Ugele B, Burckhardt G and Bahn A: Human renal organic anion transporter 4 operates as an asymmetric urate transporter. J Am Soc Nephrol 18: 430-439, 2007.
13. Bahn A, Hagos Y and Reuter S: Identification of a new urate and high affinity nicotinate transporter, hOAT10 (SLC22A13). J Biol Chem 283: 16332-16341, 2008.

14. Kimura T, Takahashi M, Yan K and Sakurai H: Expression of SLC2A9 isoforms in the kidney and their localization in polarized epithelial cells. PLoS One 9: e84996, 2014.

15. Chiba T, Matsuo H, Nagamori S, Nakayama A, Kawamura Y, Shimizu S, Sakiyama M, Hosoyamada M, Kawai S, Okada R, et al: Identification of a hypouricemia patient with SLC2A9 R380W, a pathogenic mutation for renal hypouricemia type 2. Nucleosides Nucleotides Nucleic Acids 33: 261-265, 2014.

16. Mou LJ, Jiang LP and Hu Y: A novel homozygous GLUT9 mutation cause recurrent exercise induced acute renal failure and posterior reversible encephalopathy syndrome. J Nephrol 28: 387-392, 2015.

17. Claverie-Martin F, Trujillo-Suarez J, Gonzalez-Acosta H, Aparicio C, Justa Roldan ML, Stiburkova B, Ichida K, Martín-Gomez MA, Herrero Goñi M, Carrasco Hidalgo-Barquero $\mathrm{M}$, et al: URAT1 and GLUT9 mutations in Spanish patients with renal hypouricemia. Clin Chim Acta 481: 83-89, 2018.

18. Jeannin G, Chiarelli N, Gaggiotti M, Ritelli M, Maiorca P, Quinzani S, Verzeletti F, Possenti S, Colombi M and Cancarini G: Recurrent exercise-induced acute renal failure in a young Pakistani man with severe renal hypouricemia and SLC2A9 compound heterozygosity. BMC Med Genet 15: 3, 2014.

19. Stiburkova B, Taylor J, Marinaki AM and Sebesta I: Acute kidney injury in two children caused by renal hypouricaemia type 2. Pediatr Nephrol 27: 1411-1415, 2012.

20. Ohta T, Sakano T, Igarashi T, Itami N and Ogawa T; ARF Assocoated with Renal Hypouricemia Research Group: Exercise-induced acute renal failure associated with renal hypouricaemia: Results of a questionnaire-based survey in Japan. Nephrol Dial Transplant 19: 1447-1453, 2004.

21. Erley CM, Hirschberg RR, Hoefer W and Schaefer K: Acute renal failure due to uric acid nephropathy in a patient with renal hypouricemia. Klin Wochenschr 67: 308-312, 1989.

22. Paller MS, Hoidal JR and Ferris TF: Oxygen free radicals in ischemic acute renal failure in the rat. J Clin Invest 74: 1156-1164, 1984.

23. Ohta T, Sakano T, Ogawa T, Kato J, Awaya Y, Kihara H and Kinoshita Y: Exercise-induced acute renal failure with renal hypouricemia: A case report and a review of the literature. Clin Nephrol 58: 313-316, 2002.

24. Murakami T, Kawakami H, Fukuda M and Furukawa S: Patients with renal hypouricemia are prone to develop acute renal failure-why? Clin Nephrol 43: 207-208, 1995.

25. Peden DB, Hohman R, Brown ME, Mason RT, Berkebile C, Fales HM and Kaliner MA: Uric acid is a major antioxidant in human nasal airway secretions. Proc Natl Acad Sci USA 87: 7638-7642, 1990.

26. Vollaard NB, Shearman JP and Cooper CE: Exercise-induced oxidative stress: Myths, realities and physiological relevance. Sports Med 35: 1045-1062, 2005.

27. Gofrit O, Verstandig AG and Pode D: Bilateral obstructing ureteral uric acid stones in an infant with hereditary renal hypouricemia. J Urol 149: 1506-1507, 1993.

28. Dinour D, Bahn A, Ganon L, Ron R, Geifman-Holtzman O, Knecht A, Gafter U, Rachamimov R, Sela BA, Burckhardt G and Holtzman EJ: URAT1 mutations cause renal hypouricemia type 1 in Iraqi Jews. Nephrol Dial Transplant 26: 2175-2181, 2011.

29. Bhasin B, Stiburkova B, De Castro-Pretelt M, Beck N, Bodurtha JN and Atta MG: Hereditary renal hypouricemia: A new role for allopurinol? Am J Med 127: e3-e4, 2014.

This work is licensed under a Creative Commons Attribution-NonCommercial-NoDerivatives 4.0 International (CC BY-NC-ND 4.0) License. 